CELL STRUCTURE AND FUNCTION 9, Suppl., s63-66 (1984)

(C) by Japan Society for Cell Biology

\title{
III-2 Axoplasmic Transport
}

\author{
Masanori Kurokawa \\ Department of Biochemistry, Institute of Brain Research, Faculty of \\ Medicine, University of Tokyo, Tokyo 113, Japan
}

\section{Retrograde transport}

By means of phase-contrast-cinematography, Nakai $(1955,1956,1964)$ first observed the bidirectional movement of mitochondria and lysosomes in the neurites of chick embryo sensory neurones in culture. In contrast, pinocytotic vesicles always travelled cellulipetally, i.e., from the growth cone to the perikaryon, at an average velocity of $1 \mu \mathrm{m} / \mathrm{min}$ in the cone, and of $2-5 \mu \mathrm{m} / \mathrm{min}$ after passing the cone.

A considerable portion of the rapid anterograde transport of proteins in the sensory neurones shifts over to retrograde transport (Abe, Haga and Kurokawa, 1974). Like the rapid anterograde transport, retrograde transport is also blocked by colchicine. Though the molecular species conveyed by the retrograde transport have not yet been characterised, there are indications that they differ from anterogradely transported species in some respects. After switching direction from anterograde to retrograde, a protein component with a molecular weight of $23 \mathrm{kD}$ is consistently decreased (Abe, Haga and Kurokawa, 1974). By combining immunofluorescence and immunotitration specific to dopamine- $\beta$-hydroxylase (DBH), Nagatsu and her co-workers have shown that a relatively large amount of anterogradely transported DBH is transported back to the perikaryon, but devoid of enzyme activity (Nagatsu et al., 1976). Tsukita and Ishikawa (1980) have demonstrated that the anterograde transport is mainly composed of vesicular and tubular structures, whereas the retrograde transport is composed of multivesicular and lamellar bodies.

\section{Fast transport}

Abe, Haga and Kurokawa (1973) have labelled the frog dorsal root ganglion with either ${ }_{\mathrm{L}-}\left[{ }^{14} \mathrm{C}\right]$ leucine or $\left[\mathrm{Me}^{3} \mathrm{H}\right]$ choline, or both of them, and analysed the rapid transport of proteins and phosphatidylcholine in the sciatic nerve. Identical behaviour of $\left[{ }^{14} \mathrm{C}\right]$ and $\left[{ }^{3} \mathrm{H}\right]$ labels with respect to the transport velocity, temperature dependence, blockade by colchicine, and subcellular distribution led them to conclude that the rapid anterograde transport represents a flow of membranes of microsomal nature. Tsukita and Ishikawa (1976) have subsequently shown by high-voltage electron microscopy that the smooth endoplasmic reticulum in myelinated axons constitutes a three-dimensional continuous network. There were few isolated fragments of endoplasmic reticulum and vesicles in the internodal part, whereas free vesicles were more frequently observed at the Ranvier node. They have further given morphological evidence for the involvement of smooth endoplasmic reticulum in the rapid transport (Tsukita and Ishikawa, 1979), in support of the biochemical analyses by Abe, Haga and Kurokawa (1973).

Rapid axoplasmic transport is completely blocked by low concentrations of methyl mercury, which also depolymerises microtubules in vitro (Abe, Haga and Kurokawa, 


\section{s64}

1975). This finding supports the widely accepted view that microtubules play a crucial role in the mechanism of axoplasmic transport.

Identification of individual protein species conveyed by the fast transport has been hampered mainly for technical reasons. Tashiro, Kasai and Kurokawa (1980) have found in the vegal nerve (and later in other nerves) a $26 \mathrm{kD}$ polypeptide which strongly resembles calmodulin in its chemical structure. The protein is tightly bound to the membrane, and reaches axon terminals (synaptosomes) without any apparent changes in composition. Calmodulin itself is not found in the fast transport; instead, it constitutes one of the minor proteins in the slow phase of axoplasmic transport (Tashiro and Kurokawa, 1982).

Takenaka and his colleagues developed a new technique that enables us to measure the rapid axoplasmic transport in nerves under physiological conditions. In this system, $\beta$-emission is measured with a semiconductor radiation detector (Takenaka, Horie and Sugita, 1978). The general properties of the rapid transport measured in this way agree satisfactorily with those measured by biochemical analyses (Abe, Haga and Kurokawa, 1973).

\section{Slow transport}

Slow transport amounts to two-thirds of the total axoplasmic transport. In contrast to the rapid transport which is mainly associated with membraneous organelles, slow transport is composed mainly of cytoskeletal proteins, identified as actin, tubulin, and neurofilament triplet polypeptides. The nature of the polypeptide that constitutes mammalian neurofilaments has been a matter of controversy for some years, mainly because of the contamination of glial fibrillary acidic (GFA) protein in the "neurofilament" fraction prepared by the conventional cell fractionation procedures (for review, see Kurokawa, 1981). The initial suggestion by Hoffman and Lasek (J. Cell Biol. 66. 351, 1975) that mammalian neurofilaments are composed of three polypeptides $(200 \mathrm{kD}, 160 \mathrm{kD}$ and $68 \mathrm{kD})$ migrating coherently in axons was later corroborated by the demonstration that neurofilaments purified from the sciatic nerve are composed exclusively of these three polypeptides (Mori and Kurokawa, 1979, 1980), and that degeneration of the optic nerve eliminates simultaneously the triplet polypeptides and neurofilaments, leaving the astroglial filaments intact (Tsukita, Ishikawa and Kurokawa, 1981). Astroglial filaments from the optic nerve of mature mice (and rats) consisted of two polypeptides (Tsukita, Ishikawa and Kurokawa, 1981), which were subsequently identified as GFA protein and vimentin (Yokoyama, Mori and Kurokawa, 1981).

Compositional variations in the axonal cytoskeleton are seen among nerves, as exemplified by the inability to detect the neurofilament triplet by fluorography in the vagal nerve (Tashiro, Kasai and Kurokawa, 1980; Yokoyama et al., 1980). Asymmetry of slow transport in two branches of pseudounipolar axons is unique in demonstrating compositional variations of the axonal cytoskeleton. Thus, the peak of radioactivity representing the slow transport migrates at $1 \mathrm{~mm} /$ day in the peripheral branch of the dorsal root ganglion axons, and at $0.4 \mathrm{~mm} /$ day in the central branch (Komiya and Kurokawa, 1978). Fluorographic visualisation of the slow transport has revealed that, among the cytoskeletal components, actin migrates most rapidly, the neurofilament triplet most slowly, and tubulin at an intermediate velocity (Mori, Komiya and Kurokawa, 1977). Each of actin, tubulin and the triplet migrates more rapidly in the peripheral branch than in the central branch. An even more prominent aspect of central-peripheral asymmetry relates to the tubulin/triplet or 
actin + tubulin/triplet ratio which is significantly higher in the peripheral branch, suggesting a higher growth potency of the peripheral axons (Mori, Komiya and Kurokawa, 1979).

Colchicine that completely blocks the fast (Abe, Haga and Kurokawa, 1973) and retrograde (Abe, Haga and Kurokawa, 1974) transport, does not seem to block the transport as a whole (Komiya and Kurokawa, 1980). Thus, colchicine partially blocks the transport of tubulin, but the transport of actin and the triplet does not seem to be affected. On the other hand, $\beta, \beta^{\prime}$-iminodipropionitrile (IDPN) sepcifically blocks the transport of the neurofilament triplet, without any apparent effect on the transport of tubulin and actin (Yokoyama et al., 1980), or on the fast transport (Yokoyama and Kurokawa, unpublished).

Recent development of the freeze-etch replica method in electron microscopy has thrown new light on the ultrastructure of the axonal cytoskeleton (Tsukita et al., 1982). Minor components identified in the slow transport, such as clathrin (Mori and Kurokawa, 1981) and calmodulin (Tashiro and Kurokawa, 1982) may be associated with granular structures which attach to the cytoskeletal components, and are removed by the treatment with Triton X-100 (Tsukita et al., 1982).

Intra-axonal transport of fodrin is unique in that it migrates down the axon in all velocity groups except the fast transport (Levine and Willard, J. Cell Biol. 90, $631,1981)$. An entirely different line of approach by Kakiuchi and his colleagues has identified calspectin as the major calmodulin-binding protein in the brain (Kakiuchi et al., 1982b), and the identity between fodrin and calspectin has recently been established (for review, see Kakiuchi, 1983). The mode of interaction of calspectin with calmodulin and actin has been analysed from both the biochemical and morphological viewpoints (Kakiuchi et al., 1982a; Tsukita et al., 1983).

Calcium-dependent protease that preferentially degrades the $160 \mathrm{kD}$ component of the neurofilament triplet is found associated with a cytoskeletal preparation from the rat spinal cord. The activity is dissociated from the cytoskeleton only at a high ionic strength, and can be reassociated with a protease-free cytoskeleton when the ionic strength is lowered to a physiological level (Ishizaki, Tashiro and Kurokawa, 1983). Since the $160 \mathrm{kD}$ component, together with the $200 \mathrm{kD}$ component, is believed to constitute the peripheral structure of the neurofilament, it is possible that the protease affects a subtle interaction between the neurofilament and other cytoskeletal components.

\section{REFERENCES}

Abe, T., Haga, T. and Kurokawa, M. (1973). Biochem. J. 136, 731-740.

Abe, T., Haga, T. and Kurokawa, M. (1974). FEBS Lett. 47, 272-275.

Abe, T., Haga, T. and Kurokawa, M. (1975). Brain Res. 86, 504-508.

IshizAKi, Y., TASHIro, T. and KurokaWA, M. (1983). Eur. J. Biochem. 131, 41-45.

KAKIUCHI, S. (1983). Neurochem. Int. 5, 159-169.

Kakiuchi, S., Sobue, K., Kanda, K., Morimoto, K., Tsukita, S., Tsukita, S., Ishikawa, H. and Kurokawa, M. (1982a). Biomedical Res. 3, 400-410.

Kakiuchi, S., Sobue, K., Morimoto, K. and Kanda, K. (1982b). Biochem. Int. 5, 755-762.

KomiYa, Y. and Kurokawa, M. (1978). Brain Res. 139, 354-358.

KomiYa, Y. and Kurokawa, M. (1980). Brain Res. 190, 505-516.

Kurokawa, M. (1981). Seitai-no-Kagaku 32, 19-24 (in Japanese).

Mori, H. and Kurokawa, M. (1979). Cell Struct. Funct. 4, 163-167. 
Mori, H. and Kurokawa, M. (1980). Biomedical Res. 1, 24-31.

Mori, H. and Kurokawa, M. (1981). Biomedical Res. 2, 677-685.

Mori, H., KomiYa, Y. and Kurokawa, M. (1977). Proc. Japan Acad. 53, Ser. B, 252-256.

Mori, H., Komiya, Y. and Kurokawa, M. (1979). J. Cell Biol. 82, 174-184.

Nagatsu, I., Kondo, Y., Kato, T. and Nagatsu, T. (1976). Brain Res. 116, 277-285.

NaKaI, J. (1955). Anat. Rec. 121, 462.

NAKaI, J. (1956). Amer. J. Anat. 99, 81-130.

NakaI, J. (1964). in Primitive Motile Systems in Cell Biology, eds. Allen, R. D. and Kamiya, N., pp. 377-385, Academic Press, New York.

Tashiro, T., Kasai, H. and Kurokawa, M. (1980). Biomedical Res. 1, 292-299.

Tashiro, T. and Kurokawa, M. (1982). in Axoplasmic Transport, ed. Weiss, D. G., pp. 153-160, Springer-Verlag, Berlin.

Takenaka, T., Horie, H. and Sugita, T. (1978). J. Neurobiol. 9, 317-324.

Tsukita, S. and Ishikawa, H. (1976). J. Electron Microsc. 25, 141-149.

TsukitA, S. and IshikAWA, H. (1979). Brain Res. 174, 315-318.

Tsukita, S. and Ishikawa, H. (1980). J. Cell Biol. 84, 513-540.

Tsukita, S., Ishikawa, H. and Kurokawa, M. (1981). J. Cell Biol. 88, 245-250.

Tsukita, S., Tsukita, S., Ishikawa, H., Kurokawa, M., Morimoto, K., Sobue, K. and Kakiuchi, S. (1983). J. Cell Biol. 97, 574-578.

Tsukita, S., Usukura, J., Tsukita, S. and Ishikawa, H. (1982). Neuroscience 7, 2135-2147.

Yokoyama, K., Mori, H. and Kurokawa, M. (1981). FEBS Lett. 135, 25-30.

Yokoyama, K., Tsukita, S., Ishikawa, H. and Kurokawa, M. (1980). Biomedical Res. 1, 537-547. 